\title{
Palladium Nanoparticles Catalyzed Synthesis of Benzofurans by a Domino Approach
}

Pavan Kumar Mandali

Dillip Kumar Chand*

Synthesis 2015, 47, 1661.

The melting point for compound $1 \mathrm{~m}$ was corrected to $156-158^{\circ} \mathrm{C}$ for the final print and online versions. 